\title{
Image Computation for Polynomial Dynamical Systems Using the Bernstein Expansion
}

\author{
Thao Dang and David Salinas \\ Verimag, \\ 2 avenue de Vignate, 38610 Gières, France
}

\begin{abstract}
This paper is concerned with the problem of computing the image of a set by a polynomial function. Such image computations constitute a crucial component in typical tools for set-based analysis of hybrid systems and embedded software with polynomial dynamics, which found applications in various engineering domains. One typical example is the computation of all states reachable from a given set in one step by a continuous dynamics described by a differential or difference equation. We propose a new algorithm for over-approximating such images based on the Bernstein expansion of polynomial functions. The images are stored using template polyhedra. Using a prototype implementation, the performance of the algorithm was demonstrated on two practical systems as well as a number of randomly generated examples.
\end{abstract}

\section{Introduction}

Hybrid systems, that is, systems exhibiting both continuous and discrete dynamics, have been an active research domain, thanks to their numerous applications in chemical process control, avionics, robotics, and most recently molecular biology. Due to the safety critical features of many such applications, formal analysis is a topic of particular interest. A major component in any verification algorithm for hybrid systems is an efficient method to compute the reachable sets of their continuous dynamics described by differential or difference equations. Wellknown properties of affine systems and other simpler systems can be exploited to design relatively efficient methods 1 . A recently-developed method can handle continuous systems of 100 and more variables [11. Nevertheless, non-linear systems are much more difficult to analyze.

In this work, we address the following image computation problem: given a set in $\mathbb{R}^{n}$, compute its image by a polynomial. This problem typically arises when we deal with a dynamical system of the form $x(k+1)=\pi(x(k))$ where $\pi$ is a multivariate polynomial. Such a dynamical system could result from a numerical approximation of a continuous or hybrid system. Many existing reachability computation methods for continuous systems can be seen as an extension of numerical integration. For reachability analysis which requires considering all

${ }^{1}$ The hybrid systems reachability computation literature is vast. The reader is referred to the recent proceedings of the conferences HSCC. 
possible solutions (for example, due to non-determinism in initial conditions), one has to solve the above equation with sets, that is $x(k)$ and $x(k+1)$ in the equation are subsets of $\mathbb{R}^{n}$ (while they are points if we only need a single solution, as in numerical integration). In addition, similar equations can arise in embedded control systems, such as some physical system controlled by a computer program, which is the implementation of some continuous (or possibly hybrid) controller using appropriate discretization.

Another reason for our interest in the image computation problem for polynomials is that such systems can be used to model a variety of physical phenomena in engineering, economy and bio-chemical networks. This problem was previously considered in [4, where a method using Bézier techniques from Computer Aided Geometric Design (CAGD) was proposed. The drawback of this method is that it requires expensive convex-hull and triangulation computation, which restricts its application to systems of dimensions not higher than 3,4. The essence of the new method we propose in this paper can be summarized as follows. Using a special class of polyhedra together with optimization, we are able to reduce the complexity of the required polyhedral manipulation. Furthermore, by exploiting the Bernstein expansion, we only need to solve linear programming problems instead of polynomial optimization problems.

Our method is similar to a number of existing methods for continuous and hybrid systems in the use of linear approximation. Its novelty resides in the efficient way of computing linear approximations. Indeed, a common method to approximate a non-linear function by a piecewise linear one, as in the hybridization approach [1] for hybrid systems, requires non-linear optimization. Indeed, the work presented in this paper follows the approach using template polyhedra and optimization for hybrid systems with continuous dynamics proposed in [21].

Besides constrained global optimization, other important applications of the Bernstein expansion include various control problems [7] (in particular, robust control). The approximation of the range of a multivariate polynomial over a box is also used in program analysis and optimization (for example [323]). In the hybrid systems verification, polynomial optimization is used to compute barrier certificates [18. Algebraic properties of polynomials are used to compute polynomial invariants 24] and to study the computability of image computation in [17].

The paper is organized as follows. In Section 2 we introduce the notions of template polyhedra and the Bernstein expansion. We then formally state our problem and describe an optimization-based solution. In order to transform the polynomial optimization problem to a linear programming problem, a method for computing bound functions is presented. We then describe an algorithm summarizing the main steps of our method. Some experimental results, in particular the analysis of a control and a biological systems, are reported in Section 4.

\section{Preliminaries}

Notation. Let $\mathbb{R}$ denote the set of reals. Throughout the paper, vectors are often written using bold letters. Exceptionally, scalar elements of multi-indices, 
introduced later, are written using bold letters. Given a vector $\mathbf{x}, x_{i}$ denotes its $i^{\text {th }}$ component. Capital letters, such as $A, B, X, Y$, denote matrices or sets. If $A$ is a matrix, $A^{i}$ denotes the $i^{t h}$ row of $A$. An affine function is thus represented as $\mathbf{c}^{T} \mathbf{x}+\mathbf{d}$.

We use $B_{u}$ to denote the unit box $B_{u}=[0,1]^{n}$. We use $\pi$ to denote a vector of $n$ functions such that for all $i \in\{1, \ldots, n\}, \pi_{i}$ is an $n$-variate polynomial of the form $\pi_{i}: \mathbb{R}^{n} \rightarrow \mathbb{R}$. In the remainder of the paper, we sometimes refer to $\pi$ simply as "a polynomial".

To discuss the Bernstein expansion, we use multi-indices of the form $\mathbf{i}=$ $\left(\mathbf{i}_{1}, \ldots, \mathbf{i}_{n}\right)$ where each $\mathbf{i}_{j}$ is a non-negative integer. Given two multi-indices $\mathbf{i}$ and $\mathbf{w}$, we write $\mathbf{i} \leq \mathbf{w}$ if for all $j \in\{1, \ldots, n\}, \mathbf{i}_{j} \leq \mathbf{w}_{j}$. Also, we write $\frac{\mathbf{i}}{\mathbf{w}}$ for $\left(\frac{\mathbf{i}_{1}}{\mathbf{w}_{1}}, \ldots, \frac{\mathbf{i}_{n}}{\mathbf{w}_{n}}\right)$ and $\left(\begin{array}{c}\mathbf{i} \\ \mathbf{w}\end{array}\right)$ for $\left(\begin{array}{c}\mathbf{i}_{1} \\ \mathbf{w}_{1}\end{array}\right)\left(\begin{array}{c}\mathbf{i}_{2} \\ \mathbf{w}_{2}\end{array}\right) \ldots\left(\begin{array}{c}\mathbf{i}_{n} \\ \mathbf{w}_{n}\end{array}\right)$.

Template polyhedra. A convex polyhedron is a conjunction of a finite number of linear inequalities described as $A \mathbf{x} \leq \mathbf{b}$, where $A$ is a $m \times n$ matrix, $\mathbf{b}$ is a column vector of size $m$. Template polyhedra are commonly used in static analysis of programs for computing invariants (see for example [19]). The reader is referred to [19] for a thorough description of template polyhedra.

A template is a set of linear functions over $\mathbf{x}=\left(x_{1}, \ldots, x_{n}\right)$. We denote a template by an $m \times n$ matrix $H$, such that each row $H^{i}$ corresponds to the linear function $H^{i} \mathbf{x}$. Given such a template $H$ and a real-valued vector $\mathbf{d} \in \mathbb{R}^{m}$, a template polyhedron is defined by considering the conjunction of the linear inequalities of the form $\bigwedge_{i=1, \ldots, m} H^{i} \mathbf{x} \leq d_{i}$. We denote this polyhedron by $\langle H, \mathbf{d}\rangle$.

By changing the values of the elements of $\mathbf{d}$, one can define a family of template polyhedra corresponding to the template $H$. We call $\mathbf{d}$ a polyhedral coefficient vector. Given $\mathbf{d}, \mathbf{d}^{\prime} \in \mathbb{R}^{m}$, if $\forall i \in\{1, \ldots, m\}: d_{i} \leq d_{i}^{\prime}$, we write $\mathbf{d} \preceq \mathbf{d}^{\prime}$. Given an $m \times n$ template $H$ and two polyhedral coefficient vectors $\mathbf{d}, \mathbf{d}^{\prime} \in \mathbb{R}^{m}$, if $\mathbf{d} \preceq \mathbf{d}^{\prime}$ then the inclusion relation $\langle H, \mathbf{d}\rangle \subseteq\left\langle H, \mathbf{d}^{\prime}\right\rangle$ holds, and we say that $\langle H, \mathbf{d}\rangle$ is not larger than $\left\langle H, \mathbf{d}^{\prime}\right\rangle$.

The advantage of template polyhedra over general convex polyhedra is that the Boolean operations (union, intersection) and common geometric operations can be performed more efficiently [19].

Bernstein expansion. We consider an $n$-variate polynomial $\pi: \mathbb{R}^{n} \rightarrow \mathbb{R}^{n}$ defined as: $\pi(\mathbf{x})=\sum_{\mathbf{i} \in I_{\mathbf{w}}} \mathbf{a}_{\mathbf{i}} \mathbf{x}^{\mathbf{i}}$ where $\mathbf{x}^{\mathbf{i}}=x_{1}^{\mathbf{i}_{1}} \ldots x_{n}^{\mathbf{i}_{n}}, \mathbf{a}_{\mathbf{i}}$ is a vector in $\mathbb{R}^{n} ; \mathbf{i}$ and $\mathbf{w}$ are two multi-indices of size $n$ such that $\mathbf{i} \leq \mathbf{w} ; I_{\mathbf{w}}$ is the set of all multi-indices $\mathbf{i} \leq \mathbf{w}$, that is $I_{\mathbf{w}}=\{\mathbf{i} \mid \mathbf{i} \leq \mathbf{w}\}$. The multi-index $\mathbf{w}$ is called the degree of $\pi$.

Given a set $X \subset \mathbb{R}^{n}$, the image of $X$ by $\pi$, denoted by $\pi(X)$, is defined as follows: $\pi(X)=\left\{\left(\pi_{1}(\mathbf{x}), \ldots, \pi_{n}(\mathbf{x})\right) \mid \mathbf{x} \in \mathbb{R}^{n}\right\}$.

In order to explain the Bernstein expansion of the polynomial $\pi$, we first introduce Bernstein polynomials. For $\mathbf{x}=\left(x_{1}, \ldots, x_{n}\right) \in \mathbb{R}^{n}$, the $\mathbf{i}^{\text {th }}$ Bernstein polynomial of degree $\mathbf{w}$ is: $\mathcal{B}_{\mathbf{w}, \mathbf{i}}(\mathbf{x})=\beta_{\mathbf{w}_{1}, \mathbf{i}_{1}}\left(x_{1}\right) \ldots \beta_{\mathbf{w}_{n}, \mathbf{i}_{n}}\left(x_{n}\right)$ where for a real number $y, \beta_{\mathbf{w}_{j}, \mathbf{i}_{j}}(y)=\left(\begin{array}{c}\mathbf{w}_{j} \\ \mathbf{i}_{j}\end{array}\right) y^{\mathbf{i}_{j}}\left(1-y^{\mathbf{w}_{j}-\mathbf{i}_{j}}\right)$. Then, for all $\mathbf{x} \in B_{u}=[0,1]^{n}, \pi$ can be written using the Bernstein expansion as follows: $\pi(\mathbf{x})=\sum_{\mathbf{i} \in I_{\mathbf{w}}} \mathbf{b}_{\mathbf{i}} \mathcal{B}_{\mathbf{w}, \mathbf{i}}(\mathbf{x})$ where for each $\mathbf{i} \in I_{\mathbf{w}}$ the Bernstein coefficient $\mathbf{b}_{\mathbf{i}}$ is: 


$$
\mathbf{b}_{\mathbf{i}}=\sum_{\mathbf{j} \leq \mathbf{i}} \frac{\left(\begin{array}{l}
\mathbf{i} \\
\mathbf{j}
\end{array}\right)}{\left(\begin{array}{l}
\mathbf{j} \\
\mathbf{j}
\end{array}\right)} \mathbf{a}_{\mathbf{j}} .
$$

The following property of the Bernstein coefficients is of interest. The above enclosure yields: $\forall \mathbf{x} \in B_{u}: \pi(\mathbf{x}) \in \square\left(\left\{\mathbf{b}_{\mathbf{i}} \mid \mathbf{i} \in I_{\mathbf{w}}\right\}\right)$ where $\square$ denotes the bounding box of a point set.

Let us return to the main problem of the paper, which is computing the image of a set by a polynomial. Using the above convex-hull property, we can use the coefficients of the Bernstein expansion to over-approximate the image of the unit box $B_{u}$ by the polynomial $\pi$. To compute the image of a general convex polyhedron, one can over-approximate the polyhedron by a box and then transform it to the unit box via some affine transformation. A similar idea, which involves using the coefficients of the Bézier simplex representation, was used in 4 to compute the image of a convex polyhedron. However, the convex-hull computation is expensive especially in high dimensions, which poses a major problem in continuous and hybrid systems verification approaches using polyhedral representations.

In this work, we propose a new method which can avoid complex convex-hull operations over general convex polyhedra as follows. First, we use template polyhedra to over-approximate the images. Second, the problem of computing such template polyhedra can be formulated as a polynomial optimization problem. This optimization problem is computationally difficult, despite recent progress in the development of methods and tools for polynomial programming (see for example 25136 and references therein). We therefore seek their affine bound functions for polynomials, in order to transform the polynomial optimization problem to a linear programming one, which can be solved more efficiently (in polynomial time) using well-developed techniques, such as Simplex [8] and interior point techniques [2]. Indeed, the above-described Bernstein expansion is used to compute these affine bound functions. This is discussed in the next section.

Bound functions. To compute bound functions, we employ the method using the Bernstein expansion, published in 8910]. Finding convex lower bound functions for polynomials is a problem of great interest, especially in global optimization. It is important to note that the method described in this section only works for the case where the variable domain is the unit box $B_{u}$. We however want to compute the images of more general sets, in particular polyhedra. An extension of this method to such cases will be developed in Section 3.2 .

A simple affine lower bound function is a constant function, which can be deduced from the property of the Bernstein expansion mentioned in Section 2. $x_{i} \leq \min \left\{\mathbf{b}_{\mathbf{i}} \mid \mathbf{i} \in I_{\mathbf{w}}\right\}=\mathbf{b}_{\mathbf{i}^{0}}=\mathbf{b}^{0}$. The main idea of the method is as follows. We first compute the affine lower bound function whose corresponding hyperplane passes through this control point $\mathbf{b}^{0}$. Then, we aditionally determine $(n-1)$ hyperplanes passing through $n$ other control points. This allows us to construct a sequence of $n$ affine lower bound functions $l_{0}, l_{1}, \ldots l_{n}$. We end up with $l_{n}$, a function whose corresponding hyperplane passes through a lower facet of the convex 
hull spanned by these control points. A detailed description of the algorithm can be found in [5. Note that we can easily compute upper bound functions of $\pi$ by computing the lower bound functions for $(-\pi)$ using this method and then multiply each resulting function by $(-1)$.

\section{Image Approximation Using Template Polyhedra}

We want to use a template polyhedron $\langle H, \mathbf{d}\rangle$ to over-approximate the image of a polyhedron $P$ by the polynomial $\pi$. The template matrix $H$, which is of size $m \times n$ is assumed to be given; the polyhedral coefficient vector $\mathbf{d} \in \mathbb{R}^{m}$ is however unknown. The question is thus to find $\mathbf{d}$ such that

$$
\pi(P) \subseteq\langle H, \mathbf{d}\rangle .
$$

It is not hard to see that the following condition is sufficient for (2) to hold: $\forall \mathbf{x} \in P: H \pi(\mathbf{x}) \leq \mathbf{d}$. Therefore, to determine $\mathbf{d}$, one can formulate the following optimization problem:

$$
\forall i \in\{1, \ldots, m\}, d_{i}=\max \left(\sum_{k=1}^{n} H_{k}^{i} \pi_{k}(\mathbf{x})\right) \text { subj. to } \mathbf{x} \in P .
$$

where $H^{i}$ is the $i^{\text {th }}$ row of the matrix $H$ and $H_{k}^{i}$ is its $k^{\text {th }}$ element. Note that the above functions to optimize are polynomials. As mentioned earlier, polynomial optimization is expensive. Our solution is to bound these functions with affine functions, in order to transform the above optimization problem to a linear programming one. This is formalized as follows.

\subsection{Optimization-Based Solution}

In Section 2 we discussed lower bound functions for polynomials. Note that these bound functions are valid only when the variables $\mathbf{x}$ are inside the unit box $B_{u}$. To consider more general domains, we introduce the following definition.

Definition 1 (Upper and lower bound functions). Given $f: \mathbb{R}^{n} \rightarrow \mathbb{R}$, the function $v: \mathbb{R}^{n} \rightarrow \mathbb{R}$ is called an upper bound function of $f$ w.r.t. a set $X \subset \mathbb{R}^{n}$ if $\forall \mathbf{x} \in X: f(\mathbf{x}) \leq v(\mathbf{x})$. A lower bound function can be defined similarly.

The following property of upper and lower bound functions is easy to prove.

Lemma 1. Given $X, Y \subseteq \mathbb{R}^{n}$ s.t. $Y \subseteq X$, if $v$ is an upper (lower) bound function of $f$ w.r.t. $X$, then $v$ is an upper (lower) bound function of $f$ w.r.t. $Y$.

For each $k \in\{1, \ldots, m\}$, let $u_{k}(\mathbf{x})$ and $l_{k}(\mathbf{x})$ respectively be an upper bound function and a lower bound function of $\pi_{k}(\mathbf{x})$ w.r.t. a bounded polyhedron $P \subset$ $\mathbb{R}^{n}$. We consider the following optimization problem:

$$
\forall i \in\{1, \ldots, m\}, d_{i}=\sum_{k=1}^{n} H_{k}^{i} \omega_{k} .
$$

where the term $H_{k}^{i} \omega_{k}$ is defined as follows: 
- If the element $H_{k}^{i}>0, H_{k}^{i} \omega_{k}=H_{k}^{i} \max u_{k}(\mathbf{x})$ subj. to $\mathbf{x} \in P$;

- If the element $H_{k}^{i} \leq 0, H_{k}^{i} \omega_{k}=H_{k}^{i} \min l_{k}(\mathbf{x})$ subj. to $\mathbf{x} \in P$.

The following lemma is a direct result of (4).

Lemma 2. If $\mathbf{d} \in \mathbb{R}^{m}$ satisfies (4), then $\pi(P) \subseteq\langle H, \mathbf{d}\rangle$.

Proof. It is indeed not hard to see that the solution $d_{i}$ of the optimization problems (4) is greater than or equal to the solution of (3). Hence, if $\mathbf{d}$ satisfies (4), then $\forall i \in\{1, \ldots, m\} \forall \mathbf{x} \in P: \sum_{k=1}^{n} H_{k}^{i} \pi_{k}(\mathbf{x}) \leq d_{i}$. This implies that $\forall \mathbf{x} \in P: H \pi(\mathbf{x}) \leq \mathbf{d}$, that is the image $\pi(P)$ is included in $\langle H, \mathbf{d}\rangle$.

We remark that if all the bound functions in (41) are affine and $P$ is a bounded convex polyhedron, $\mathbf{d}$ can be computed by solving at most $2 n$ linear programming problems. It remains now to find the affine bound functions $u_{k}$ and $l_{k}$ for $\pi$ w.r.t. a polyhedron $P$, which is the problem we tackle in the next section.

\subsection{Computing Affine Bound Functions over Polyhedral Domains}

As mentioned earlier, the method to compute affine bound functions for polynomials in Section 2 can be applied only when the function domain is a unit box, anchored at the origin. The reason is that the expression of the control points of the Bernstein expansion in (11) is only valid for this unit box. If we overapproximate $P$ with a box $B$, it is then possible to derive a formula expressing the Bernstein coefficients of $\pi$ over $B$. However, this formula is complex and its representation and evaluation can become expensive.

We alternatively consider the composition of the polynomial $\pi$ with an affine transformation $\tau$ that maps the unit box $B_{u}$ to $B$. The functions resulting from this composition are still polynomials, for which we can compute their bound functions over the unit box. This is explained more formally in the following.

Let $B$ be the bounding box of the polyhedron $P$, that is, the smallest box that includes $P$. The composition $\gamma=(\pi o \tau)$ is defined as $\gamma(\mathbf{x})=\pi(\tau(\mathbf{x}))$. The functions $\tau$ and $\gamma$ can be computed symbolically, which will be discussed later.

Lemma 3. Let $\gamma=\pi$ o $\tau$. Then, $\pi(P) \subseteq \gamma\left(B_{u}\right)$.

Proof. By the definition of the composition $\gamma, \gamma\left(B_{u}\right)=\left\{\pi(\tau(\mathbf{x})) \mid \mathbf{x} \in B_{u}\right\}$. Additionally, $\tau\left(B_{u}\right)=B$. Therefore, $\gamma\left(B_{u}\right)=\pi(B)$. Since the polyhedron $P$ is included in its bounding box $B$, we thus obtain $\pi(P) \subseteq \pi(B)=\gamma\left(B_{u}\right)$.

We remark that the above proof is still valid for any affine function $\tau$. This means that instead of an axis-aligned bounding box, we can over-approximate $P$ more precisely with an oriented (i.e. non-axis-aligned) bounding box. This can be done using the following method.

\subsection{Computing an Oriented Bounding Box}

The directions of an oriented bounding box can be computed using Principal Component Analysis [14. We first choose a set $S=\left\{\mathbf{s}^{1}, \mathbf{s}^{2}, \ldots, \mathbf{s}^{m}\right\}$ of $m$ point: 2

${ }^{2}$ By abuse of notation we use $m$ to denote both the number of template constraints and the number of points here. 
in the polyhedron $P$, such that $m \geq n$. We defer a discussion on how this point set is selected to the end of this section. PCA is used to find an orthogonal basis that best represents the point set $S$. More concretely, we use $\overline{\mathbf{s}}$ to be the mean of $S$, that is $\overline{\mathbf{s}}=\frac{1}{m} \sum_{i=1}^{m} \mathbf{s}^{i}$ and we denote $\tilde{\mathbf{s}}_{i, j}=\mathbf{s}_{i}^{j}-\overline{\mathbf{s}}_{i}$. For two points $\mathbf{s}^{i}$ and $\mathbf{s}^{j}$ in $S$, the covariance of their translated points is: $\operatorname{cov}\left(\mathbf{s}_{i}, \mathbf{s}_{j}\right)=\frac{1}{m-1} \sum_{k=1}^{m} \tilde{\mathbf{s}}_{k, i} \tilde{\mathbf{s}}_{k, j}$. Then, we define the co-variance matrix $C$ such that the element $C_{i j}=\operatorname{cov}\left(\mathbf{s}^{i}, \mathbf{s}^{j}\right)$. The $n$ largest singular values of $C$ provide the orientation of the bounding box. More concretely, since $C$ is symetric, by singular value decomposition, we have $C=U \Lambda U^{T}$ where $\Lambda$ is the matrix of singular values. The axes of the bounding box are hence determined by the first $n$ columns of the matrix $U$, and its centroid is $\overline{\mathbf{s}}$.

We now discuss how to select the set $S$. When the vertices of $P$ are available, we can include them in the set. However, if $P$ is given as a template polyhedron, this requires computing the vertices which is expensive. Moreover, using only the vertices, when their distribution do not represent the geometric form of the polyhedron, may cause a large approximation error, since the resulting principal directions are not the ones along which the points inside $P$ are mostly distributed. To remedy this, we sample points inside $P$ as follows. First, we compute an axis-aligned bounding box of $P$ (this can be done by solving $2 n$ linear programming problems). We then uniformly sample points inside this bounding box and keep only the points that satisfy the constraints of $P$. Uniform sampling on the boundary of $P$ in general enables a better precision. More detail on this can be found in [5].

\subsection{Image Computation Algorithm}

The following algorithm summarizes the main steps of our method for overapproximating the image of a bounded polyhedron $P \subset \mathbb{R}^{n}$ by the polynomial $\pi$. The templates are an input of the algorithm. In the current implementation of the algorithm, the templates can be fixed by the user, or the templates forming regular sets are used.

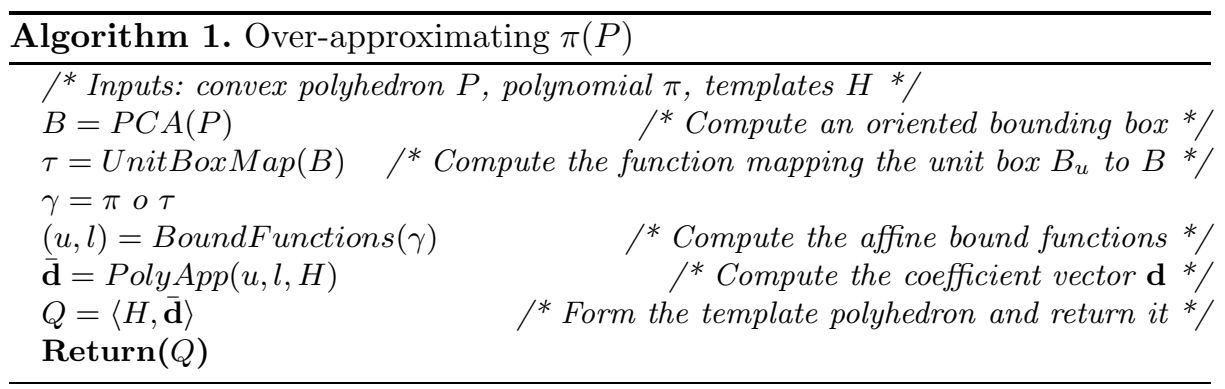

The role of the procedure $P C A$ is to compute an oriented bounding box $B$ that encloses $P$. The procedure UnitBoxMap is then used to determine the affine function $\tau$ that maps the unit box $B_{u}$ at the origin to $B$. This affine function 
is composed with the polynomial $\pi$, the result of which is the polynomials $\gamma$. The affine lower and upper bound functions $l$ and $u$ of $\gamma$ are then computed, using the Bernstein expansion. The function PolyApp determines the polyhedral coefficient vector $\mathbf{d}$ by solving the linear programs in (4) with $u, l$ and the optimization domain is $B_{u}$. The polyhedral coefficient vector $\mathbf{d}$ are then used to define a template polyhedron $Q$, which is the result to be returned.

Based on the analysis so far, we can state the correctness of Algorithm 1

Theorem 1. Let $\langle H, \overline{\mathbf{d}}\rangle$ be the template polyhedron returned by Algorithm 1 . Then $\pi(P) \subseteq\langle H, \overline{\mathbf{d}}\rangle$.

We remark that $u$ and $l$ are upper and lower bound functions of $\gamma$ with respect to $B_{u}$. It is not hard to see that $\tau^{-1}(P) \subseteq B_{u}$ where $\tau^{-1}$ is the inverse of $\tau$. Using the property of bound functions, $u$ and $l$ are also bound functions of $\gamma$ with respect to $\tau^{-1}(P)$. Hence, if we solve the optimization problem over the domain $\tau^{-1}(P)$ (which is often smaller than $B_{u}$ ), using Lemma 2, the resulting polyhedron is still an over-approximation of $\pi(P)$. This remark can be used to obtain more accurate results.

Approximation errors and Complexity. We finish this section by briefly discussing the precision and complexity of our method. A more detailed analysis can be found in 5. The approximation errors are caused by the use of bound functions, the bounding box approximation and template polyhedra.

It can be proven that in one dimensional cases, the error between the bound functions and the original polynomial is quadratic in the length of box domains. This quadratic convergence seems to hold for higher dimensional cases in practice, as shown in 9. We conjecture that there exists a subdivision method of the box $B$ which allows a quadratic convergence of the error. This subdivision method is similar to the one used for finding roots of a polynomial with quadratic convergence 16 .

On the other hand, a polyhedron can be approximated by a set of nonoverlapping oriented boxes with arbitrarily small error. Then, for each box, we compute a bounding function, with which we then compute a coefficient for each template. Finally, for each template, we take the largest coefficient to define the template polyhedron. Since the boxes are smaller, the bounding functions are more precise, we can thus improve the coefficients as much as desired.

Concerning the error inherent to the approximation by template polyhedra, it can be controlled by fine-tuning the number of template constraints. If using this method with a sufficient number of templates to assure the same precision as the convex hull in our previous Bézier method 4, then the convergence of both methods are quadratic. However the Bezier method requires expensive convexhull and triangulation operations, and geometric complexity of resulting sets may grow step after step. Combining template polyhedra and bounding functions allows a good accuracy-cost compromise.

We now discuss the complexity of our algorithm. Let each polynomial $\pi_{i}$ be written as $\pi_{i}=\sum_{\mathbf{j} \in I_{i}} a_{\mathbf{j}}^{i} \mathbf{x}^{\mathbf{j}}$ where each $a_{\mathbf{j}}^{i} \neq 0$. We denote by $\#\left(\pi_{i}\right)$ the number 
of such monomials in $\pi_{i}$, i.e. the cardinality of $I_{i}$. Let $K$ be the maximal number of monomials in each $\pi_{i}$, that is $K=\max _{i \in\{1, \ldots, n\}} \#\left(\pi_{i}\right)$.

First, we remark that the computation of the bound functions and PCA only requires manipulating matrices and linear equations. Additionally, linear programming with $n$ variables and $m$ constraints can be solved in polynomial time $O\left((m n)^{3.5}\right)$.

The proofs of the following results can be found in [5]. The complexity of the computation of the bound functions is $\mathcal{O}\left(n^{4}+K n^{5}\right)$. The complexity of the computation of an affine function $\tau$ mapping the unit box to an oriented box is $\mathcal{O}\left(n n^{3.5}\right)$ (due to $n$ LP problems). The approximation using a template polyhedron requires solving $2 n$ LP problems over the unit box and has thus the complexity $\mathcal{O}\left(2 n(2 n n)^{3.5}\right)$ (see (4) ).

The exponentiel factor in the complexity of our algorithm comes from the composition of $\pi$ and an affine transformation $\tau$. Let us suppose that we use a simple composition algorithm whose complexity depends on the number of monomials 3 . The following theorem shows some cases for which our algorithm has a polynomial time complexity.

Theorem 2. If $\pi$ and $\tau$ satisfy two conditions:

$$
\begin{aligned}
& \text { (1) } \forall i \in\{1, \ldots, n\}: \sum_{\mathbf{j} \in I_{i}} \sum_{k=1}^{n} \mathbf{j}_{k}=O(\ln (n)) \\
& \text { (2) } \forall i \in\{1, \ldots, n\} \#\left(\tau_{i}\right) \leq 2
\end{aligned}
$$

then the composition $\pi$ o $\tau$ has in total $\mathcal{O}\left(K n^{3}\right)$ monomials, thus the computation of $\pi$ o $\tau$ can be done in $\mathcal{O}\left(K n^{3}\right)$.

The proof of this can be found in [5. Note that if we use axis-aligned bounding boxes, each component of $\tau$ always have 2 terms, and the second condition of the theorem are satisfied. However, this polynomial time complexity w.r.t. the dimension may not hold if we use oriented bounding boxes to over-approximate the reachable sets before mapping them to the unit box. Indeed, in this case each component of $\tau$ may have more than 2 terms.

Concerning the complexity w.r.t. the number of iterations, if the number of template constraints is constant, we can prove that the complexity depends linearly on the number of iterations (see more in [5]).

\section{Experimental Results}

We have implemented our method in a prototype tool using the template polyhedral library developed by S. Sankaranarayanan [20] and the library lpsolve for linear programming. In the following, we demonstrate the method with two examples: a control system (modelled as a hybrid system) and a biological system (modelled as a continuous system). The time efficiency of the tool is also evaluated by considering using a number of randomly generated polynomials.

\footnotetext{
${ }^{3}$ Advanced composition algorithms, e.g. 22, can achieve a better complexity.
} 
A control system. The control system we consider is the Duffing oscillator [156]. Its continuous-time dynamics is described by $\ddot{y}(t)+2 \zeta \dot{y}(t)+y(t)+$ $y(t)^{3}=u(t)$, where $y \in \mathbb{R}$ is the state variable and $u \in \mathbb{R}$ is the control input. The damping coefficient $\zeta=0.3$. In [6], using a forward difference approximation with a sampling period $h=0.05$, this system is approximated by the following discrete-time model: $x_{1}(k+1)=x_{1}(k)+h x_{2}(k), x_{2}(k+1)=$ $\left.\left.-h x_{1}(k)+(1-2 \zeta h) x_{2}(k)+h u\right) k\right)-h x_{1}(k)^{3}$.

In [6], an optimal predictive control law $u(k)$ was computed by solving a parametric polynomial optimization problem. In Figure 4 one can see the phase portrait of the system under this control law and without it (i.e. $\forall k g e 0 u(k)=0$ ) is shown. We model this control law by the following switching law with 3 modes: $u(k)=0.5 k$ if $0 \leq k \leq 10, u(k)=5-0.5(k-10) / 3$ if $10<k \leq 40$, and $u(k)=0$ if $k>40$. The controlled system is thus modelled as a hybrid automaton with 3 discrete modes. The result obtained using our tool on this system is shown in Figure 4, which is coherent with the phase portrait in 6]. The initial set is a ball with radius $1 e-04$. The number of template constraints is 100 . In addition to the reachable set after 120 steps (computed after $3 s$ ), in Figure 4, we also illustrate the approximation error by visusalizing the template polyhedron after the first step and a cloud of exact points (obtained by sampling the initial set and applying the polynomial to the sampled points).

A biological system. The second example is the well-known Michaelis-Menten enzyme kinetics [12, where $E$ is the concentration of an enzyme that combines with a substrate $S$ to form an enzyme substrate complex $E S$. In the next step, the complex can be dissociated into $E$ and $S$ or it can further proceed to form a product $P$.

This pathway kinetics can be described by the following ODEs where $x_{1}, x_{2}$, $x_{3}$ and $x_{4}$ are the concentrations of $S, E, E S$ and $P: \dot{x}_{1}=-\theta_{1} x_{1} x_{2}+\theta_{2} x_{3}$,
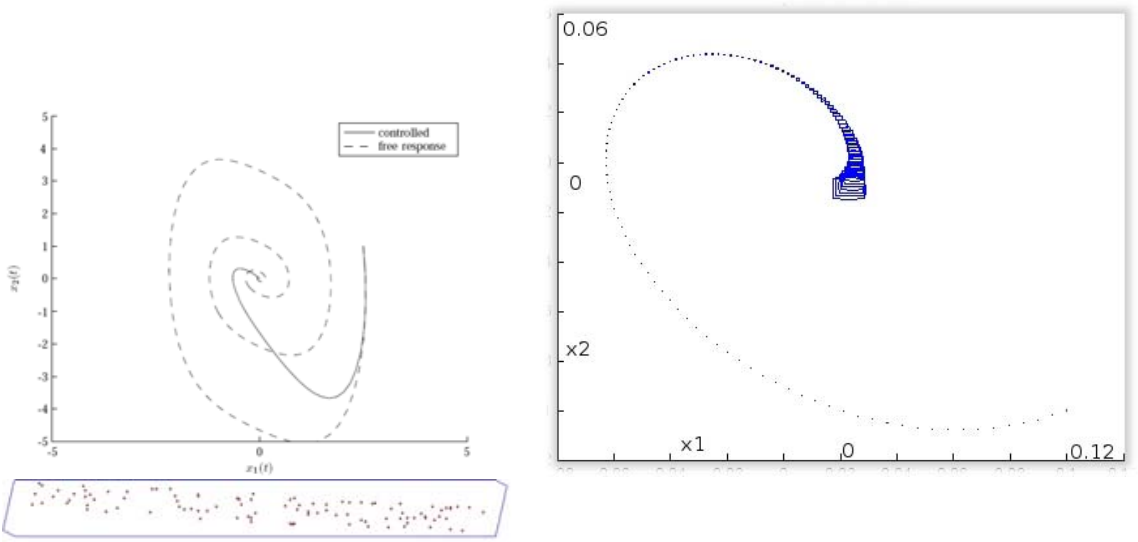

Fig. 1. The Duffing oscillator: phase portrait, the reachable set, and the reachable set after the first step 

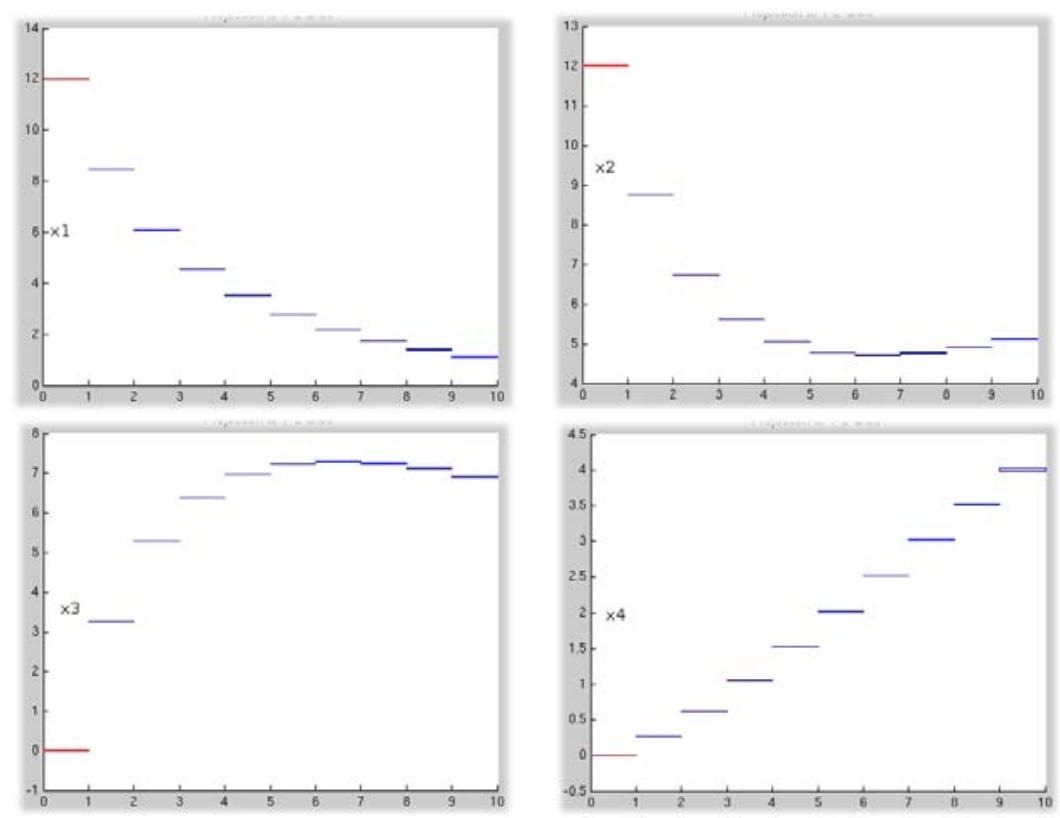

Fig. 2. Michaelis-Menten enzyme kinetics. The evolution of the reachable set after 10 steps.

$\dot{x}_{2}=-\theta_{1} x_{1} x_{2}+\left(\theta_{2}+\theta_{3}\right) x_{3}, \dot{x}_{3}=\theta_{1} x_{1} x_{2}+\left(\theta_{2}+\theta_{3}\right) x_{3}, \dot{x}_{4}=\theta_{3} x_{3}$. Using a second order Runge Kutta discretization with time step 0.3, we obtain

$$
\begin{aligned}
\pi_{1}(\mathbf{x}) & =x_{1}-0.053838 x_{1} x_{2}+0.001458 x_{1}^{2} x_{2}+0.001458 x_{1} x_{2}^{2}-3.9366 e-5 . x_{1}^{2} x_{2}^{2} \\
& +0.005775 x_{3}-0.002025 x_{1} x_{3}-0.000162 x_{2} x_{3}+5.9049 e-5 x_{1} x_{2} x_{3}-6.075 e-6 x_{3}^{2} \\
\pi_{2}(\mathbf{x}) & =x_{2}-0.051975 x_{1} x_{2}+0.001458 x_{1}^{2} x_{2}+0.001458 x_{1} x_{2}^{2}-3.9366 e-5 x_{1}^{2} x_{2}^{2}+0.0721875 x_{3} \\
& -0.002025 x_{1} x_{3}-0.000162 x_{2} x_{3}+5.9049 e-5 x_{1} x_{2} x_{3}-6.075 e-6 x_{3}^{2} \\
\pi_{3}(\mathbf{x}) & =0.051975 x_{1} x_{2}-0.001458 . x 1^{2} x_{2}-0.001458 x_{1} x_{2}^{2}+3.9366 e-5 x 1^{2} x_{2}^{2} \\
& +0.927812 x_{3}+0.002025 x_{1} x_{3}+0.000162 x_{2} x_{3}-5.9049 e-5 x_{1} x_{2} x_{3}+6.075 e-6 x_{3}^{2} \\
\pi_{4}(\mathbf{x}) & =0.001863 x_{1} x_{2}+0.0664125 x_{3}+x_{4} .
\end{aligned}
$$

The reachable set computed for all the initial states inside a ball centered at $(12,12,0,0)$ with radius $1 e-0.4$ is shown in Figure 2. The number of template constraints is 60 . In order to compare with the result in 12, the figures depict the evolution of each variable for the first 10 steps (the horizontal axis is time). In the vertical axis, the minimal and maximal values of the variables are shown. This result is conherent with the simulation result in [12. The computation time for 20 steps is $3.7 \mathrm{~s}$.

Randomly generated systems. In order to evaluate the performance of our method, we tested it on a number of randomly generated polynomials in various dimensions and maximal degrees (the maximal degree is the largest degree for 


\begin{tabular}{|c|c|c|c|c|}
\hline $\operatorname{dim}$ & degree & & $\mathrm{nb}$ steps & nb constraints $=5 \times \mathrm{dim}$ \\
\hline 2 & & 2 & $10.02 \mathrm{~s}$ & 10 \\
\hline 2 & & 2 & $100.18 \mathrm{~s}$ & \\
\hline 2 & & 2 & $1001.82 \mathrm{~s}$ & \\
\hline 2 & & 3 & $10.01 \mathrm{~s}$ & \\
\hline 2 & & 3 & $100.32 \mathrm{~s}$ & \\
\hline 2 & & 3 & $1001.98 \mathrm{~s}$ & \\
\hline 3 & & 2 & $10.01 \mathrm{~s}$ & 15 \\
\hline 3 & & 2 & $100.37 \mathrm{~s}$ & \\
\hline 3 & & 2 & $1003.64 \mathrm{~s}$ & \\
\hline 3 & & 3 & $10.02 \mathrm{~s}$ & \\
\hline 3 & & 3 & $100.3 \mathrm{~s}$ & \\
\hline 3 & & 3 & $1003.84 \mathrm{~s}$ & \\
\hline 4 & & 2 & 10035 & 20 \\
\hline $\begin{array}{l}4 \\
4\end{array}$ & & 2 & $100.6 \mathrm{~s}$ & 20 \\
\hline 4 & & 2 & $1006.64 \mathrm{~s}$ & \\
\hline 4 & & 3 & $10.06 \mathrm{~s}$ & \\
\hline 4 & & 3 & $100.67 \mathrm{~s}$ & \\
\hline 4 & & 3 & $1006.41 \mathrm{~s}$ & \\
\hline
\end{tabular}

Fig. 3. Computation time for randomly generated polynomial systems

\begin{tabular}{|c|c|c|c|}
\hline $\operatorname{dim}$ & degree & nb steps & nb constraints $=5 \times \mathrm{dim}$ \\
\hline 5 & 2 & $10.12 \mathrm{~s}$ & 25 \\
\hline 5 & 2 & $101.02 \mathrm{~s}$ & \\
\hline 5 & 2 & $10010.2 \mathrm{~s}$ & \\
\hline 5 & 3 & $10.37 \mathrm{~s}$ & \\
\hline 5 & 3 & $101.22 \mathrm{~s}$ & \\
\hline 5 & 3 & $10010.74 \mathrm{~s}$ & \\
\hline 6 & 2 & $10.61 \mathrm{~s}$ & 30 \\
\hline 6 & 2 & $102.01 \mathrm{~s}$ & \\
\hline 6 & 2 & $10016.85 \mathrm{~s}$ & \\
\hline 6 & 3 & $13.99 \mathrm{~s}$ & \\
\hline 6 & 3 & $1042.47 \mathrm{~s}$ & \\
\hline 6 & 3 & $100400.30 s$ & \\
\hline 7 & 2 & $14.11 \mathrm{~s}$ & 35 \\
\hline 7 & 2 & $1060.12 \mathrm{~s}$ & \\
\hline 7 & 2 & $100568.35 \mathrm{~s}$ & \\
\hline 7 & 3 & $130.01 \mathrm{~s}$ & \\
\hline 7 & 3 & $10345.68 s$ & \\
\hline 7 & 3 & $1003856.4 \mathrm{~s}$ & \\
\hline
\end{tabular}

Fig. 4. Computation time for randomly generated polynomial systems

all variables). For a fixed dimension and degree, we generated different examples to estimate an average computation time. In the current implementation, polynomial composition is done symbolically, and we do not yet exploit the possibility of sparsity of polynomials (in terms of the number of monomials). The computation time shown in Figures 3, 4 does not include the time for polynomial composition. Note that the computation time for 7 -variate polynomials of degree 3 is significant, because the randomly generated polynomials have a large number of monomials; however, practical systems often have a much smaller number of monomials. As expected, the computation time does not grows linearly w.r.t. 
the number of steps. This can be explained by the use of template polyhedra where the number of constraints can be chosen according to required precisions and thus control better the complexity of the polyhedral operations, compared to general convex polyhedra. Indeed, when using general polyhedra, the operations such as convex hull may increase their geometric complexity (roughly described by the number of vertices and constraints).

Conclusion. In this paper we propose a new method to compute images of polynomials. This method combines the ideas from optimization and the Bernstein expansion. This result can be readily applicable to solve the reachability analysis problem for hybrid systems with polynomial continuous dynamics.

The performance of the method was demonstrated on a number of randomly generated examples, which shows an improvement in efficiency compared to our previously developed method using Bézier techniques 4. These encouraging results also show an important advantage of the method: thanks to the use of template polyhedra as a symbolic set representations, the complexity and precision of the method are more controllable than those using general polyhedra.

There are a number interesting directions to explore. Indeed, different tools from geometric modeling could be exploited to improve the efficiency of the method. For example, polynomial composition can be done for sparse polynomials more efficiently using the blossoming technique [22]. In addition to more experimentation on other hybrid systems case studies, we intend to explore a new application domain, which is verification of embedded control software. In fact, multivariate polynomials arise in many situations when analyzing programs that are automatically generated from practical embedded controllers.

Acknowledgement. We would like to thank S. Sankaranarayanan, F. Ivancic and O. Maler for inspiring us and for their valuable collaboration.

\section{References}

1. Asarin, E., Dang, T., Girard, A.: Hybridization methods for the analysis of nonlinear systems. Acta Informatica 43(7), 451-476 (2007)

2. Boyd, S., Vandenberghe, S.: Convex optimization. Cambridge Uni. Press, Cambridge (2004)

3. Clauss, F., Yu, C.I.: Application of symbolic approach to the Bernstein expansion for program analysis and optimization. Program. Comput. Softw. 30(3), 164-172 (2004)

4. Dang, T.: Approximate reachability computation for polynomial systems. In: Hespanha, J.P., Tiwari, A. (eds.) HSCC 2006. LNCS, vol. 3927, pp. 138-152. Springer, Heidelberg (2006)

5. Dang, T., Salinas, D.: Computing set images of polynomias. Technical report, VERIMAG (June 2008)

6. Fotiou, I.A., Rostalski, P., Parrilo, P.A., Morari, M.: Parametric optimization and optimal control using algebraic geometriy methods. Int. Journal of Control 79(11), 1340-1358 (2006)

7. Garloff, J.: Application of Bernstein expansion to the solution of control problems. University of Girona, pp. 421-430 (1999) 
8. Garloff, J., Jansson, C., Smith, A.P.: Lower bound functions for polynomials. Journal of Computational and Applied Mathematics 157, 207-225 (2003)

9. Garloff, J., Smith, A.P.: An improved method for the computation of affine lower bound functions for polynomials. In: Frontiers in Global Optimization. Series Nonconvex Optimization and Its Applications. Kluwer Academic Publ., Dordrecht (2004)

10. Garloff, J., Smith, A.P.: A comparison of methods for the computation of affine lower bound functions for polynomials. In: Jermann, C., Neumaier, A., Sam, D. (eds.) COCOS 2003. LNCS, vol. 3478, pp. 71-85. Springer, Heidelberg (2005)

11. Girard, A., Le Guernic, C., Maler, O.: Efficient Computation of Reachable Sets of Linear Time-Invariant Systems with Inputs. In: Hespanha, J.P., Tiwari, A. (eds.) HSCC 2006. LNCS, vol. 3927, pp. 257-271. Springer, Heidelberg (2006)

12. He, F., Yeung, L.F., Brown, M.: Discrete-time model representation for biochemical pathway systems. IAENG Int. Journal of Computer Science 34(1) (2007)

13. Henrion, D., Lasserre, J.B.: Gloptipoly: Global optimization over polynomials with matlab and sedumi. In: Proceedings of CDC (2002)

14. Jolliffe, I.T.: Principal Component Analysis. Springer, Heidelberg (2002)

15. Jordan, D.W., Smith, P.: Nonlinear Ordinary Differential Equations. Oxford Applied Mathematics and Computer Science. Oxford Uni. Press, Oxford (1987)

16. Mourrain, B., Pavone, J.P.: Subdivision methods for solving polynomial equations. Technical report, INRIA Research report, 5658 (August. 2005)

17. Platzer, A., Clarke, E.M.: The Image Computation Problem in Hybrid Systems Model Checking. In: Bemporad, A., Bicchi, A., Buttazzo, G. (eds.) HSCC 2007. LNCS, vol. 4416, pp. 473-486. Springer, Heidelberg (2007)

18. Prajna, S., Jadbabaie, A.: Safety verification of hybrid systems using barrier certificates. In: Alur, R., Pappas, G.J. (eds.) HSCC 2004. LNCS, vol. 2993, pp. 477-492. Springer, Heidelberg (2004)

19. Sankaranarayanan, S., Sipma, H., Manna, Z.: Scalable analysis of linear systems using mathematical programming. In: Cousot, R. (ed.) VMCAI 2005. LNCS, vol. 3385, pp. 25-41. Springer, Heidelberg (2005)

20. Sankaranarayanan, S.: Mathematical analysis of programs. Technical report, Standford, PhD thesis (2005)

21. Sankaranarayanan, S., Dang, T., Ivancic, F.: Symbolic Model Checking of Hybrid Systems using Template Polyhedra. In: Ramakrishnan, C.R., Rehof, J. (eds.) TACAS 2008. LNCS, vol. 4963, pp. 188-202. Springer, Heidelberg (2008)

22. Seidel, H.-P.: Polar forms and triangular B-spline surfaces. In: Blossoming: The New Polar-Form Approach to Spline Curves and Surfaces, SIGGRAPH 1991 (1991)

23. Tchoupaeva, I.: A symbolic approach to Bernstein expansion for program analysis and optimization. In: Duesterwald, E. (ed.) CC 2004. LNCS, vol. 2985, pp. 120-133. Springer, Heidelberg (2004)

24. Tiwari, A., Khanna, G.: Nonlinear systems: Approximating reach sets. In: Alur, R., Pappas, G.J. (eds.) HSCC 2004. LNCS, vol. 2993, pp. 600-614. Springer, Heidelberg (2004)

25. Vandenberghe, S., Boyd, S.: Semidefinite programming. SIAM Review 38(1), 49-95 (1996) 